\title{
Depression, Anxiety, and Stress among Senior High School Students in Selected Private Schools
}

\author{
Junnecca J. Lagahit \\ Fellowship Baptist College, Kabankalan City, Philippines \\ junnelagahit@yahoo.com
}

\begin{abstract}
This descriptive-correlational study aimed to determine the prevalence of depression, anxiety, and stress among three hundred twenty (320) senior high school students in selected private schools in Kabankalan City, Negros Occidental, Philippines when they are taken as a whole and grouped according to sex, career track, living arrangement and family monthly income. Specifically, it aimed to determine if there is a significant relationship between the level of depression, anxiety, and stress, and demographic profile of the senior high school students. The data were gathered through the Depression, Anxiety, and Stress Scale (DASS-21). The results of the study revealed that there were significant relationships among the selected demographics, namely, between living arrangement and anxiety, and between sex and stress. Results have an implication on the practice of guidance and counseling and in mental health practice. The findings of this study provided baseline information for an enhanced guidance program.
\end{abstract}

Keywords: Senior High School Students, Depression, Anxiety, Stress, Enhanced Guidance Program, Kabankalan City, Philippines 